\title{
Enhancements of Reliability Centered Maintenance analysis and its application to the railway industry
}

\author{
Roberto Mascherona \\ Aramis s.r.l., Milano, Italy.E-mail: roberto.mascherona@aramis3d.com
}

Luca Bellani

Aramis s.r.l., Milano, Italy.E-mail: luca.bellani@aramis3d.com

Michele Compare

Aramis s.r.l., Milano, Italy. E-mail: michele.compare@aramis3d.com

Energy Department, Politecnico di Milano, Italy.

Roberto Trucco

ALSTOM Ferroviaria S.p.A, Savigliano (CN), Italy.E-mail: roberto.trucco@alstomgroup.com

\author{
Enrico Zio \\ Energy Department, Politecnico di Milano, Italy.E-mail: enrico.zio@polimi.it \\ Aramis s.r.l., Milano, Italy \\ MINES ParisTech, PSL Research University, CRC, Sophia Antipolis, France \\ Eminent Scholar, Department of Nuclear Engineering, College of Engineering, Kyung Hee University, \\ Republic of Korea
}

Reliability Centered Maintenance (RCM) is a mature technique for effective maintenance decision making in complex systems. Although RCM is widely used in industry, there are major limiting factors that prevent its wider application such as the lack of reliability data at the beginning of the system operating life, the inaccuracy of the supplier data, the focus of the analysis on the failure modes rather than on the component and the fact that economic considerations do not enter the RCM process. We propose an approach to address all these issues, based on the integration of RCM with system Life Cycle Cost (LCC) models. This allows factoring a cost-benefit analysis into the technical considerations, thus yielding the optimal final maintenance decision. A practical example from ALSTOM is shown, in which, for confidentiality, the model parameter values have been opportunely modified.

Keywords: Reliability Centered Maintenance, Life Cycle Cost, Railway industry.

\section{INTRODUCTION}

Reliability Centered Maintenance (RCM) Nowlan and Heap (1978); Rausand (1998); Rausand and Vatn (2008) was proposed in the 1970's to effectively manage maintenance in complex systems. Nowadays, RCM is standardized for the different industrial sectors IEC 60300-3-11 (2009); SAE JA1012 (2002); MIL-STD-2173 (1986); NAVAIR 00-25-403 (2005); USACERL TR 99/41 (1999) and it is supported by the availability of advanced Computerized Maintenance Management Systems (CMMSs) Rastegari and Mobin (2016); Wienker et al. (2016); Carretero et al. (2003), with many success cases reported Nowlan and Heap (1978); Moubray (1997).

The main idea of RCM is to concentrate the maintenance efforts on the components of the asset most critical for safety and business, and apply to them the most effective maintenance approach, as resulting from the analysis of their reliability characteristics Rausand (1998).

In spite of the maturity of RCM, still there are some issues preventing its wider application to industry. First, data to infer the component reliability behavior are often lacking. For example, this is the case of the early operating life of a new technology system or a subsystem provided by a new supplier: the data gathered from the previous version may be not straightforwardly applicable to the new one. Yet, the reliability data provided by the suppliers can be inaccurate. For instance, data are often collected in operating conditions significantly different from those actually experienced by the system or through laboratory test campaigns performed in unrealistic ground benign

Proceedings of the 30th European Safety and Reliability Conference and the 15th Probabilistic Safety Assessment and Management Conference Edited by Piero Baraldi, Francesco Di Maio and Enrico Zio Copyright (C) ESREL2020-PSAM15 Organizers.Published by Research Publishing, Singapore. 
environments. Moreover, it is the authors' experience in the railway industry that some suppliers give only a failure rate value to represent the component reliability behavior. This entails that they implicitly assume a memoryless exponential behavior for the component failure time and, thus, they do not consider the other possible evolutions of the hazard rate such as the wear-out or infant mortality phases of the well-known bathtub curve. Finally, in some cases the estimation of the failure rate can be even deliberately adjusted to increase the benefit for the suppliers. For example, in case the supplier has the ownership of the maintenance service, it could be convenient for them to declare a failure rate value larger than that really estimated, because this reduces the risk of paying penalties whenever the reliability performance are worsen than that agreed in the contract.

Another limiting factor for a wider RCM application is that the selection of the maintenance strategy is guided towards the solution that is the best from the technical point of view, only. Indeed, the final maintenance decision heavily depends also on economic aspects. For this, RCM must be integrated with Life Cycle Cost (LCC) models that estimate the costs of operating and maintaining the component over its lifetime Campbell and Jardine (2001). This allows factoring a costbenefit analysis into the technical considerations, to yield the optimal final maintenance decision.

Finally, RCM selects the best maintenance strategy for each Failure Mode (FM); nonetheless, the optimization of the settings of the selected maintenance strategies must consider all the component FMs.

In this work, we propose a methodology to overcome all the mentioned RCM limiting factors (i.e., lack of reliability data, memoriless assumption, final decisions not integrating LCC analysis outcomes), to extend its application to complex systems at the beginning of their operational life.

The proposed methodology is applied to a practical example in the railway industry, although it can be applied also to other industrial sectors. For confidentiality, this example is slightly modified with respect to the real one, and the values considered are for illustration, only.

The paper is organized as follows. Section 2 briefly illustrates the RCM approach. In Section 3, we describe the knowledge, information and data typically available at the beginning of the system operating life. Section 4 proposes a decision model for a component with hidden and operation FMs, for which the RCM procedure has selected scheduled mainteanance. This model is applied to a condenser motor of a Heating, Ventilation and Air Conditioning (HVAC) system of train saloon in Section 5. Section 6 concludes the work.

\section{RCM at a glance}

Whichever the RCM reference standard is, the underlying idea of RCM is to first identify the Maintenance Critical Items (MCI), which impact most on safety, environment and business. This allows concentrating the efforts to carry out the time-costly RCM on the parts of the asset which are supposed to benefit most from a revision of the maintenance approach. After that, a Failure Mode and Effect Analysis (FMEA) is performed to analyze the Failure Modes (FMs) of these MCI and, thus, identify those most relevant (often refered to as dominant FMs), which the maintenance efforts must be concentrated on.

To find the best maintenance approach for every dominant FM, RCM relies on a decision flowchart, which can be adapted to the specific industrial needs. We consider the general flowchart in Figure 1.

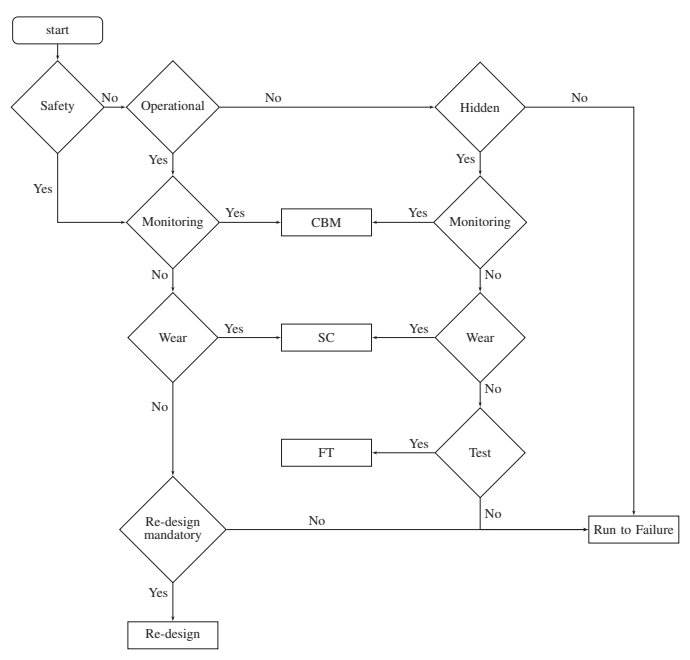

Fig. 1.: RCM Decision Tree

The first question is about the effect of the analyzed FM, which can be classified as:

- Safety $(\mathrm{S})$, if the FMEA indicates that the FM has non-negligible direct consequences on safety or environment.

- Operational (O); every FM entailing relevant impacts on system reliability or availability undergoes a detailed analysis of its maintenance strategy.

- Hidden $(\mathrm{H})$; contrarily to the 'Evident" failures, the consequences of hidden FMs become evident only when other combined failures occur. These FMs are usually linked to items that perform functions related to security, protection, re- 
serve (redundancy) and control, which need the additional failure of those item that are being secured, protected, redounded and controlled to become evident. Although not directly affecting the operability of the system, Hidden FMs can increase the risk of operational FMs.

If none of these three questions is affirmatively answered, then the FM can be managed through the run-to-failure approach. Otherwise, whichever the decision tree branch is (i.e., $\mathrm{S}, \mathrm{O}$ or $\mathrm{H}$ ), the second question is about the possibility of monitoring any physical variable indicative of the FM degradation and defining a threshold value for it, at which performing maintenance can avoid the component failure IEC 60300-3-11 (2009); SAE JA1012 (2002). In case of affirmative answer, Condition-Based Maintenance (CBM, e.g., Zio and Compare (2013)) is considered technically feasible; otherwise, the decision flowchart proceeds with other questions about the reliability characteristics of the component to check the applicability of scheduled maintenance. The rationale of this question is that if failures are random (i.e., obeying the exponential distribution), then preventive maintenance approaches are useless. In case of hidden FMs, functional checks can be performed: the larger their frequency, the larger the component availability Zio (2007). If these preventive approaches are not applicable either, the component is inevitably run to failure and taken care of by corrective maintenance.

In this work, we disregard the first branch of the tree, i.e., safety, and develop a LCC model for components with both operational and hidden FMs. The LCC analysis goal is, then, to select the optimal interval times for each scheduled activity and for the functional tests.

\section{Knowledge, Information and Data available for RCM}

At the beginning of the operating life of a complex system, reliability data of its subsystems may be lacking and the only pieces of information available are those from the supplier, who usually provides the FMEA together with the values of the component failure rates and indications of the intervals for the scheduled maintenance actions. To formalize this, we assume that every component is given a constant failure rate $\lambda^{s}(t)=\lambda^{s}$ by the supplier; the component is affected by $M$ different FMs, which are assumed statistically independent on each other. To link the component failure rate, $\lambda^{s}$, to the frequency of its $M$ FMs we rely on the following model:

$$
\lambda_{m}^{s}=\lambda^{s} \cdot p_{m}
$$

where $p_{m}$ is the portion of occurrence of failure mode $m \in\{1, \ldots, M\}$ with respect to the total number of component failures: $\lambda^{s}=\sum_{m=1}^{M} \lambda_{m}^{s}$ This modeling approach is justified by the theory of Poisson processes Papoulis and Pillai (2002).

On the other side, the assumption of constant failure rate does not allow identifying the opportunities to change the maintenance strategy or the scheduled maintenance times, which is at the heart of the RCM process. To do this, we must consider the possible wear-out and infant mortality behaviors that the component could experience when operated. These behaviors can be described by the Weibull distribution, which is often used in industrial practice due to its flexibility: it allows modeling hazard functions both increasing and decreasing over time, with different speeds Lai and Xie (2006).

The hazard rate of the $m$-th FM of the component obeying a Weibull distribution reads:

$$
\lambda_{m}^{w b}(t)=\frac{\beta_{m}}{\alpha_{m}}\left(\frac{t}{\alpha_{m}}\right)^{\beta_{m}-1}
$$

where $\alpha_{m}$ and $\beta_{m}$ are the scale and shape paremeters, repsectively, whetreas $t$ is the time from component installation.

To work with a Weibull distribution coherent with the information available about the constant failure rate of each FM, we assume that it has been estimated as the average number of FMs experienced within the scheduled maintenance time interval $\tau_{m}^{s}$ suggested by the supplier Zio (2007). This average number must be the same for the two distributions:

$$
\int_{0}^{\tau_{m}^{s}} \lambda_{m}^{w b}(t) d t=\left(\frac{\tau_{m}^{s}}{\alpha_{m}}\right)^{\beta_{m}}=\lambda_{m}^{s} \cdot \tau_{m}^{s}
$$

Equation 3 encodes two unknown parameters $\alpha_{m}$ and $\beta_{m}$, which are linked by the following relation:

$$
\alpha_{m}=\frac{\left(\tau_{m}^{s}\right)^{1-1 / \beta_{m}}}{\left(\lambda_{m}^{s}\right)^{1 / \beta_{m}}}
$$

We indicate by $\boldsymbol{\beta}=\left[\beta_{1}, \ldots, \beta_{M}\right] \in \mathcal{B}$ the vector containing the shape parameters of all FMs affecting the component. Given the lack of reliability data, we assume that the shape parameter, $\beta_{m}$, ranges in interval $\left[\beta_{m}, \overline{\beta_{m}}\right]$, which depends on the specific application, $m \in\{1, \ldots, M\}$ : $\beta_{m}<1$ is suitable for components with expected

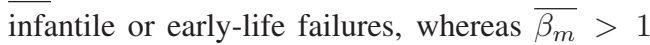
indicates that component ware-out is expected: the larger its value, the faster the degradation. This way, we consider the uncertainty on the possible failure behaviors of the FMs, including that of a fast acceleration of ware-out after $\tau_{m}$, if we set $\overline{\beta_{m}}>>1$.

To consider the possible uncertainty in the failure 
rate values provided by the suppliers, we assume that the actual failure frequency $\lambda_{m} \in\left[\lambda_{m}, \overline{\lambda_{m}}\right]$, where $\lambda_{m}=l \cdot \lambda_{m}^{s}$ and $\overline{\lambda_{m}}=g \cdot \lambda_{m}^{s}, 0<l<g$. The $\mathrm{D} \overline{\mathrm{M}}$ can set $l>1$ if the failure rate value provided by the supplier is considered underestimated, whereas $l<1$ corresponds to the situation where the DM feels that the supplier estimation is conservative.

We indicate by $\boldsymbol{\lambda}=\left[\lambda_{1}, \ldots, \lambda_{M}\right]$ the vector containing the failure rates of all FMs affecting the component.

\section{Decision model for components undergoing scheduled actions and functional checks}

Assume that the FMECA of a component has identified $k<M$ hidden FMs and $M-k$ operational FMs, for which the RCM decision tree has led to select the scheduled maintenance approach. Without loss of generality, we indicate the hidden FMs as $\mu=1, \ldots, k$, whereas the operational FMs are $m=k+1, \ldots, M$.

Upon the occurrence of a hidden FM $\mu \in$ $\{1, \ldots, k\}$, the failure rate of the $m$-th operational FM increases by a factor $\chi_{m, \mu}>1, m=k+$ $1, \ldots, M$.

If we indicate by $X_{\mu}$ the binary variable such that $X_{\mu}=1$ when FM $\mu$ has occurred and $X_{\mu}=0$, otherwise, then, the effects of hidden FMs on operational FM $m \in\{\mu+1, \ldots, M\}$ can be considered through one of the following additive or multiplicative, respectively, updating models:

$$
\begin{gathered}
\lambda_{m} \leftarrow\left(1+\sum_{\mu=1}^{k}\left(\chi_{m, \mu}-1\right) \cdot X_{\mu}\right) \cdot \lambda_{m} \\
\lambda_{m} \leftarrow \prod_{\mu=1}^{k}\left(1+\left(\chi_{m, \mu}-1\right) \cdot X_{\mu}\right) \cdot \lambda_{m}
\end{gathered}
$$

To prevent FMs $m=\mu+1, \ldots, M$, the component undergoes scheduled actions. These always leave the component in As Good As New (AGAN) conditions.

Let $\tau=\left[\tau_{\mu+1}, \ldots, \tau_{M}\right] \in \mathcal{T}$ be the vector of the corresponding maintenance periods. The number of scheduled maintenance actions for FM $m=$ $\mu+1, \ldots, M$ performed over the time horizon $\Omega$ is $N_{m} \leq\left\lfloor\frac{\Omega}{\tau_{m}}\right\rfloor$. This number is a random variable, which depends on the component failure behavior. If we indicate by $c_{m}^{p}$ the fixed cost of a single preventive maintenance task $m=\mu+1, \ldots, M$, the total fixed cost for scheduled maintenance over the time horizon reads:

$$
C_{m}^{p}=N_{m} \cdot c_{m}^{p}
$$

The average total time to perform scheduled maintenance action for preventing FM $m=\mu+$ $1, \ldots, M$ over $\Omega$ reads:

$$
T_{m}^{p}=N_{m} \cdot T T R_{m}^{p}
$$

where $T T R_{m}^{p}$ is the average time to perform the single scheduled action. Possible synergies can be easily encoded in this scheme.

To detect hidden FMs, we assume that the component undergoes a single test that can detect all its hidden FMs. This test is performed every $\pi$ units of time; then, the related total fixed cost $C^{\pi}$ and total time to perform tests $T^{\pi}$ read:

$$
C^{\pi}=N_{\pi} \cdot c_{\pi} \quad T^{\pi}=N_{\pi} \cdot T T R_{\pi}
$$

where $N_{\pi}$ is the random number of tests performed within time horizon $\Omega$, whereas $c_{\pi}$ is the costs of test performed and TTR $R_{\pi}$ is the time to perform the single test.

The total cost for corrective maintenance over the system time horizon reads:

$$
C^{f}=N^{\Omega} \cdot c^{f}
$$

where $c^{f}$ is the expected cost of the single corrective action, whichever the $\mathrm{FM}$, whereas $N^{\Omega}$ is the number of failures of the component within the operating time horizon $\Omega$. This is a random variable depending on the Weibull distributions of the component FMs, test interval $\pi$ and vector $\tau$ : larger intervals between tests delay the detection of hidden FMs and, thus, increase the time window in which the failure rates of the operational FMs are larger than in nominal conditions. Similarly, larger scheduled intervals $\tau$ entail longer ware out. Thus, increments in $\pi$ and $\tau$ lead to larger expected numbers of failures $N^{\Omega}$. On the other hand, larger values of $\pi$ and of the values in $\tau$ allow decreasing the expected numbers $N^{\pi}$ and $N_{m}$ of test and preventive actions, respectively, to be performed over the train operating time horizon.

We indicate by $T T R^{f}$ the expected time to perform a single corrective action; then, the total time spent within time horizon $\Omega$ to perform corrective actions reads:

$$
T^{f}=N^{\Omega} \cdot T T R^{f}
$$

The $L C C$ of the component reads:

$$
\begin{aligned}
& L C C(\boldsymbol{\tau}, \pi, \beta, \lambda)=C^{f}+T^{f} \cdot H^{f} \\
& +\sum_{m=\mu+1}^{M}\left(C_{m}^{p}+T_{m}^{p} \cdot H_{m}^{p}\right)+\left(C^{\pi}+T^{\pi} \cdot H^{\pi}\right)
\end{aligned}
$$

where $H^{\pi}$ is the hourly cost of test, whereas $H^{f}$ and $H_{m}^{p}$ are the hourly cost of corrective and scheduled maintenance on operational FM $m=$ $\mu+1, \ldots, M$, respectively. These depend on the 
number of workers required to perform the action and their skills.

Notice that the hourly cost $H_{m}^{p}$ can also be set to zero, as for example in the case where there is a diagnostic system.

Notice also that Equation 12 also encodes the costs related to the maintenance actions performed upon a negative test: these are corrective maintenance actions, whose costs are accounted by the first two addends.

Equation 12 allows finding the optimal values of $\tau$ and $\pi$ yielding the minimum LCC over the whole life $\Omega$ of the system. For this, we append $\pi$ to $\tau$ in vector $\boldsymbol{d}=[\boldsymbol{\tau}, \pi]$ and propose the following procedure:

- Consider a grid of values of $\boldsymbol{d}$;

- For every value of $\tau$ and $\pi$, consider a grid of values of $\boldsymbol{\beta}$ and $\boldsymbol{\lambda}$ and calculate the corresponding $\boldsymbol{\alpha}$ (Equation 4);

- Apply Monte Carlo simulation to estimate $L C C(\boldsymbol{d}, \boldsymbol{\beta}, \boldsymbol{\lambda})$

- Use the pairwise dominance criteria Salo and Hämäläinen (1995) to eliminate the maintenance times $\boldsymbol{d}$ yielding dominated $L C C$ values: $\boldsymbol{d}^{x}$ is pairwise dominated by $\boldsymbol{d}^{y}, \boldsymbol{d}^{x} \prec_{p} \boldsymbol{d}^{y}$ if $\forall \boldsymbol{\beta} \in \mathcal{B} \forall \boldsymbol{\lambda} \in \mathcal{L}$ :

$$
L C C\left(\boldsymbol{d}^{x}, \boldsymbol{\beta}, \boldsymbol{\lambda}\right)>L C C\left(\boldsymbol{d}^{y}, \boldsymbol{\beta}, \boldsymbol{\lambda}\right)
$$

The pairwise dominance is a sufficient condition fo the absolute dominance Salo and Hämäläinen (1995): $\boldsymbol{d}^{x}$ is absolutely dominated by $\boldsymbol{d}^{y}, \boldsymbol{d}^{x} \prec \boldsymbol{d}^{y}$ if:

$$
\min _{\boldsymbol{\beta}, \boldsymbol{\lambda}} L C C\left(\boldsymbol{d}^{x}, \boldsymbol{\beta}, \boldsymbol{\lambda}\right)>\max _{\boldsymbol{\beta}, \boldsymbol{\lambda}} L C C\left(\boldsymbol{d}^{y}, \boldsymbol{\beta}, \boldsymbol{\lambda}\right)
$$

If the portfolio of non dominated solutions contains more than one element, the decision on the optimal time to perform maintenance is based on the DM preferences. For illustration, a risk averse DM who wants to select the optimal policy that minimizes the $L C C$ of the worst combination of parameters (i.e., maximin regret policy Salo and Hämäläinen (1995)) operates as follows:

- Conservatively assume that the total LCC following scheduled maintenance policy and test performed at times $\tau$ and $\pi$ is that obtained under the combination of parameters yielding the maximum expected cost: $L C C(\boldsymbol{d})=$ $\max _{\boldsymbol{\beta}, \boldsymbol{\lambda}} L C C(\boldsymbol{d}, \boldsymbol{\beta}, \boldsymbol{\lambda})$

- The optimal scheduled maintenance and test times $\tau^{*}$ and $\pi^{*}$ are those minimizing the LCC, i.e., $\boldsymbol{d}^{*}=\left[\boldsymbol{\tau}^{*}, \pi^{*}\right]=$ arg $\min _{\boldsymbol{d}} L C C(\boldsymbol{d})$.

Finally notice that $k=M$ is typical of auxiliary components (e.g., sensors) which allow detecting and/or preventing failure of other components. Thus, these components need to be considered together with those which their failure mitigation applies to.

\section{Example of application}

Consider the condenser motor of a Heating Ventilation and Air Conditioning (HVAC) system for train saloon. There are $M=3 \mathrm{FMs}$, including $\mu=1$ hidden FM:

- $F M_{1}$ : bearings degradation. This hidden FM causes over-vibration, which increases the frequency of the other two FMs. We assume $\beta_{1} \in[1.1,1.5]$. The RCM suggets a periodical functional check.

- $F M_{2}$ : Coil in short-circuit. This leads to the HVAC out of service. A corrective approach has been indicated by RCM for this FM. We set $\chi_{2,1}=2.5$ and $\beta_{2} \in$ $[1.1,1.5]$.

- $F M_{3}$ : internal mechanical breakage. This entails the HVAC out of service. The RCM analysis has shown that a scheduled maintenance (component replacement or overhaul) is doable to prevent this FM. We set $\chi_{3,1}=2.5$ and $\beta_{3} \in[1.5,3.5]$.

The portions of occurrence of each of them are $p_{1}=0.25, p_{2}=0.30$ and $p_{3}=0.45$. In Table 1 , the LCC parameters are summarized. These are derived from the values provided by the supplier, which, for confidentility, have been opportunely re-scaled.

To select the scheduled maintenance frequency yielding the minimum LCC, we apply the approach described in Section 4, which yields $\boldsymbol{d}^{*}=$ $[1,31,16]$.

To justify this result, in Figure 2 we fix $\pi=1$ (i.e., functional test performed every year), $\tau_{2}=31$, i.e., corrective maintenance, and show the total maintenance costs as a function of the time interval $\tau_{3}$ between consecutive scheduled actions on $F M_{3}$. We consider $l=1$ and $g=2$, whereby $\lambda_{m} \in\left[\lambda_{m}=\lambda^{s} \cdot p_{m}, \overline{\lambda_{m}}=2 \cdot \lambda^{s} \cdot p_{m}\right]$. We show the results for the two extreme values of the interval (i.e., $\lambda^{s}$ and $2 \cdot \lambda^{s}$ ), reported in different colors and markers.

Notice that $\tau_{3}=10$ years is the maintenance period proposed by the item supplier, whereas $\tau_{3}=31$ years corresponds to performing corrective actions only.

The two curves with the same color represent the upper (continuous line) and lower (dotted line) bounds of the maintenance costs for all possible combinations of Weibull parameters $\boldsymbol{\alpha}, \boldsymbol{\beta}$. From the analysis of Figure 2, we can see that: 
Table 1.: LCC parameters

\begin{tabular}{|c|c|c|c|c|c|c|c|c|c|}
\hline$\lambda^{s}\left[\mathrm{~h}^{-1}\right]$ & $c^{f}[€]$ & $T T R^{f}[\mathrm{~h}]$ & $c_{3}^{p}[€]$ & $T T R_{3}^{p}[\mathrm{~h}]$ & $H^{f}=H_{3}^{p}=H^{\pi}[€ / \mathrm{h}]$ & $c^{\pi}[€]$ & $T T R^{\pi}[\mathrm{h}]$ & $\Omega$ [years] & functioning h/y \\
\hline $2.6 \cdot 10^{-6}$ & 390 & 2.3 & 75 & 3 & 70 & 0 & 0 & 31 & 7300 \\
\hline
\end{tabular}

- For all values of $\boldsymbol{\lambda}$, the expected maintenance cost strongly increases with the number of performed scheduled tasks: $\tau_{3}=10$ years entails a total of 3 scheduled actions, resulting in the most expensive maintenance strategy. Similarly, $\tau_{3} \in[11,15]$ years entails 2 scheduled actions and, finally, $\tau_{3} \in[16,30]$ years entails a single action.

- For all values of $\boldsymbol{\lambda}$, the maintenance cost monotonically increases over the time intervals $\tau_{3} \in[11,15]$ years $(2$ scheduled tasks) and $\tau_{3} \in[16,30]$ years (one scheduled tasks).

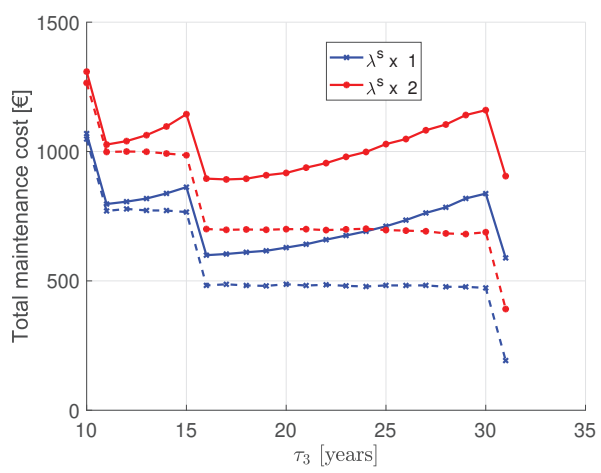

Fig. 2.: Upper and lower bounds of total maintenance cost vs scheduled maintenance time of $F M_{3}$ for different values of $\lambda^{s}$

If we apply the maximin regret policy Salo and Hämäläinen (1995), we have to refer to the upper curve, which reaches the smallest cost value at $\tau_{3}=16$ years.

Figure 3 represents the expected number of failures $N^{\Omega}$ over the values of $\tau_{3}$ for $\lambda=\lambda^{s}, 2 \lambda^{s}$. It can be seen that corrective maintenance policy entails $N^{\Omega}$ up to three times larger than that corresponding to the policy suggested by the supplier. For $\tau_{3}=16$ years, in the worst case $\left(\lambda=2 \lambda^{s}\right)$ the number of expected failures increases up to $25 \%$.

\section{CONCLUSIONS AND FURTHER DEVELOPMENTS}

We have shown an approach to integrate RCM with system Life Cycle Cost (LCC) models, which allows factoring a cost-benefit analysis into the technical considerations to yield the optimal

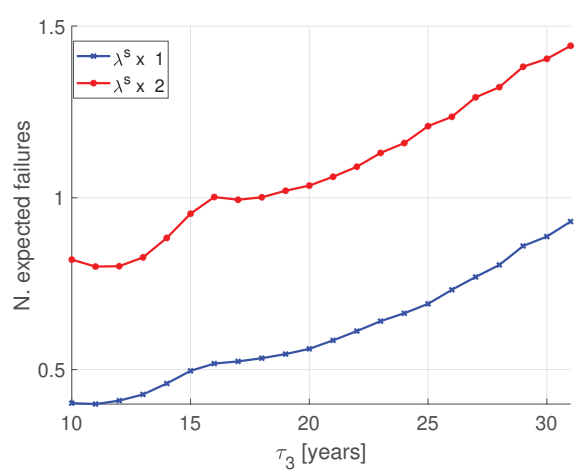

Fig. 3.: Maximum expected number of failures vs scheduled maintenance time of $F M_{3}$ for different values of $\lambda^{s}$

final maintenance decision. The proposed approach extends the applicability of RCM in that it overcomes some main limitations such as the lack of reliability data at the beginning of the system operating life, the inaccuracy of the supplier data, the focus of the analysis on the failure modes rather than on the component.

The models proposed can be further refined depending on the availability of additional information, either gathered from expert-based knowledge or from data.

For instance, to decrease the epistemic uncertainty in the values of the parameters $\alpha_{m}$ and $\beta_{m}$, i.e., to overcome the assumption that all $\beta_{m}$ distributions are uniform in their domain, more realistic distributions $\pi\left(\beta_{m}\right)$ of $\beta_{m}$ can be inferred. This allows distinguishing between failure modes leading to early failures (i.e., $\pi\left(\beta_{m}\right)$ has larger mass on values of $\beta_{m}<1$ ) from those due to aging effects (i.e., $\pi\left(\beta_{m}\right)$ has larger mass on values of $\beta_{m}>1$ ); depending on the total amount of information provided, a distribution can be used to model also the uncertainty in the parameters $\alpha_{m}$, thus getting rid of some of the assumptions made in the FMECA.

Furthemore, the derived distributions of $\alpha_{m}$ and $\beta_{m}$ can be embedded in a Bayesian framework as prior distributions for future analyses: additional collected data can be used to derive the posterior distributions. The procedure is iterated every time new data are collected, thus allowing more accurate $L C C$ estimations. 


\section{References}

Campbell, J. D. \& A. K. S. Jardine (2001). Maintenance excellence : optimizing equipment lifecycle decisions. New York : Marcel Dekker.

Carretero, J., J. M. Pérez, F. García-Carballeira, A. Calderón, J. Fernández, J. D. García, A. Lozano, L. Cardona, N. Cotaina, \& P. Prete (2003). Applying $\mathrm{rcm}$ in large scale systems: a case study with railway networks. Reliability Engineering \& System Safety 82(3), 257 - 273.

IEC 60300-3-11 (2009). Dependability management - Part 3-11: Application Guide - Reliability Centered Maintenance. International Electrotechnical Commission.

Lai, C.-D. \& M. Xie (2006). Stochastic ageing and dependence for reliability. Springer.

MIL-STD-2173 (1986). Reliability-Centered Maintenance. Requirements for Naval Aircraft, Weapon Systems and Support Equipment. U.S. Department of Defense, Washington, DC.

Moubray, J. (1997). Reliability Centered Maintenance ( $2^{\text {nd }}$ ed.). NY: Industrial Press.

NAVAIR 00-25-403 (2005). Guidelines for the naval aviation reliability-centered maintenance process. U.S. Naval Air Systems Command.

Nowlan, F. \& H. Heap (1978). Reliability Centered Maintenance. Technical report.

Papoulis, A. \& S. U. Pillai (2002). Probability, Random Variables, and Stochastic Processes (4 ed.). McGraw-Hill Higher Education.

Rastegari, A. \& M. Mobin (2016). Maintenance decision making, supported by computerized maintenance management system. Proceedings of the Annual Reliability and Maintainability Symposium. art. no .7448086.

Rausand, M. (1998). Reliability Centered Maintenance. Reliability Engineering and System Safety 60, 121-132.

Rausand, M. \& J. Vatn (2008). Reliability Centred Maintenance. Springer Series in Reliability Engineering 8, 79-108.

SAE JA1012 (2002). , A Guide to the ReliabilityCentered Maintenance (RCM) Standard. Society of Automotive Engineers.

Salo, A. \& R. Hämäläinen (1995). Preference programming through approximate ratio comparisons. European Journal of Operational Research 82(3), 458-475.

USACERL TR 99/41 (1999). Reliability centered maintenance (RCM) guide. Operating a more effective maintenance program. U.S. Army Corps of Engineers.

Wienker, M., K. Henderson, \& J. Volkerts (2016). The computerized maintenance management system an essential tool for world class maintenance. Procedia Engineering 138, 413-420.

Zio, E. (2007). An introduction to the basics of reliability and risk analysis, Volume 13 . World scientific.
Zio, E. \& M. Compare (2013). Evaluating maintenance policies by quantitative modeling and analysis. Reliability Engineering and System Safety 109, 53-65. 